\title{
Usefulness of IgM-specific enzyme immunoassays for serodiagnosis of syphilis: comparative evaluation of three different assays
}

\author{
Bosshard, Philipp P
}

\begin{abstract}
OBJECTIVES: IgM antibodies are usually the first to be produced during treponemal infection. Three commercially available enzyme immunoassays (EIA) for detection of IgM antibodies against Treponema pallidum were evaluated. METHODS: Results of the Anti-Treponema-pallidumELISA (IgM; Euroimmun), Pathozyme Syphilis M Capture (Omega Diagnostics) and recomWell Treponema IgM (Mikrogen) were compared with those of the T. pallidum particle agglutination (TPPA) and the Venereal Disease Research Laboratory (VDRL) tests for 307 serum samples. RESULTS: The overall sensitivity (95\% confidence interval [CI]) of the TPPA was 100\% (97.7-100\%) compared to $83.3 \%$ (76.5-88.8\%) of the VDRL, 88.5\% (82.4-93.0\%) of the Pathozyme, 84.6\% (78.0-89.9) of the Euroimmun, and $73.6 \%(66.1-80.4 \%)$ of a modified recomWell test procedure. Specificities were in the range of 91.4$100 \%$. In primary syphilis, sensitivities of the Pathozyme (89.8\%; 95\% CI, 79.2-96.2\%) and Euroimmun tests $(81.4 \%$; $95 \%$ CI, $69.1-90.3 \%)$ were significantly higher $(\mathrm{p}<0.05)$ than the sensitivity of the VDRL test $(61 \% ; 95 \%$ CI, 47.4-73.5\%). IgM EIAs even were positive in some cases of suspected very early infection where the VDRL was non-reactive and the TPPA was indeterminate. CONCLUSIONS: In cases of suspected early infection specific IgM EIAs should be used in addition to other screening tests. The VDRL is not recommended for screening.
\end{abstract}

DOI: https://doi.org/10.1016/j.jinf.2013.03.011

Posted at the Zurich Open Repository and Archive, University of Zurich

ZORA URL: https://doi.org/10.5167/uzh-85204

Journal Article

Accepted Version

Originally published at:

Bosshard, Philipp P (2013). Usefulness of IgM-specific enzyme immunoassays for serodiagnosis of syphilis: comparative evaluation of three different assays. Journal of Infection, 67(1):35-42.

DOI: https://doi.org/10.1016/j.jinf.2013.03.011 
1 Usefulness of IgM-specific enzyme immunoassays for serodiagnosis

2 of syphilis: comparative evaluation of three different assays

3

4

$5 \quad$ Philipp P. Bosshard

6

7 Dept. of Dermatology, University Hospital Zurich, Zürich, Switzerland

8

9 Running title

10 Evaluation of three IgM-EIAs for diagnosis of syphilis

11

12 Corresponding author

13 Philipp P. Bosshard

14 Department of Dermatology

15 Zurich University Hospital

16 Gloriastrasse 31

$17 \mathrm{CH}-8091$ Zürich

18 Switzerland

19 Tel + 41442553972

$20 \quad F a x+41442554418$

21 philipp.bosshard@usz.ch

22

23 Keywords

24 Syphilis, serology, IgM, ELISA, EIA, diagnosis

25

26 Word count

27 Manuscript (excluding abstract, tables, acknowledgment and references): 2570 28 


\section{Summary}

30 Objectives. IgM antibodies are usually the first to be produced during treponemal infection.

31 Three commercially available enzyme immunoassays (EIA) for detection of IgM antibodies

32 against Treponema pallidum were evaluated.

33 Methods. Results of the Anti-Treponema-pallidum-ELISA (IgM; Euroimmun), Pathozyme

34 Syphilis-M Capture (Omega Diagnostics) and recomWell Treponema IgM (Mikrogen) were

35 compared with those of the Treponema pallidum particle agglutination (TPPA) and the Venereal

36 Disease Research Laboratory (VDRL) tests for 307 serum samples.

37 Results. The overall sensitivity (95\% confidence interval [CI]) of the TPPA was $100 \%$ (97.7-

$38100 \%)$ compared to $83.3 \%(76.5-88.8 \%)$ of the VDRL, $88.5 \%(82.4-93.0 \%)$ of the Pathozyme,

$3984.6 \%(78.0-89.9)$ of the Euroimmun, and $73.6 \%(66.1-80.4 \%)$ of a modified recomWell test

40 procedure. Specificities were in the range of $91.4-100 \%$. In primary syphilis, sensitivities of the

41 Pathozyme (89.8\%; 95\% Cl, 79.2-96.2\%) and Euroimmun tests (81.4\%; 95\% Cl, 69.1-90.3\%)

42 were significantly higher $(p<0.05)$ than the sensitivity of the VDRL test $(61 \% ; 95 \% \mathrm{Cl}, 47.4-$

$4373.5 \%)$. IgM EIAs even were positive in some cases of suspected very early infection where the

44 VDRL was non-reactive and the TPPA was indeterminate.

45 Conclusions. In cases of suspected early infection specific IgM EIAs should be used in addition

46 to other screening tests. The VDRL is not recommended for screening.

47

48

49 Keywords

50 Syphilis; serology; IgM; ELISA; EIA; diagnosis; Treponema pallidum 


\section{Introduction}

53 Serology remains the mainstay of laboratory detection of Treponema pallidum infections. Two

54 classes of antibody tests are available; treponemal and non-treponemal. In Europe, it is recommended to use a treponemal antigen test as a single screening test, e.g. an enzyme immunoassay (EIA) or the Treponema pallidum particle agglutination test (TPPA), ${ }^{1}$ with positive samples being confirmed by a second treponemal test. Non-treponemal tests, e.g. VDRL (Venereal Disease Research Laboratory) or RPR (Rapid Plasma Reagin), are useful for detection of active infection and for monitoring treatment response, ${ }^{2}$ however, they are less specific than treponemal tests and lack sensitivity in primary and late syphilis patients. ${ }^{2-5}$ Additionally, they are labour intensive and cannot be run on automated platforms. $\lg \mathrm{M}$ class antibodies are usually the first to be produced during treponemal infection. ${ }^{6}$ It has been shown that IgM-specific EIAs are very sensitive in detecting primary and secondary syphilis ${ }^{2,4,5,7-10}$ with the IgM EIA being the first test positive in some instances. It is recommended to request a specific anti-treponemal lgM test if primary syphilis is suspected. ${ }^{1,2}$ Although many different commercial IgM EIA tests are available, few have been analysed for their performance at different stages of syphilis, namely the Mercia Syphilis M EIA (Microgen Products Ltd) $)^{4,5,8-12}$ the Eti-syphilis-M (DiaSorin) ${ }^{7}$ and the recomWell Treponema IgM (Mikrogen). ${ }^{13}$ Additionally, the Mercia Syphilis M EIA has been evaluated for diagnosing maternal and congenital syphilis ${ }^{14}$ and the use of Euroimmun EIA and Western-blot IgG and IgM assays for screening blood donors has been investigated. ${ }^{15}$ In the present study, three commercially available EIAs to detect IgM antibodies to $T$. pallidum were evaluated (Anti-Treponema-pallidum-ELISA IgM, Euroimmun; Pathozyme Syphilis M Capture, Omega Diagnostics; recomWell Treponema IgM, Mikrogen). Sensitivities were determined with consecutive sera from untreated syphilis patients at different disease stages. Specificities were analysed by a panel of sera including sera of patients with diseases potentially interfering with syphilis serology tests. 
80 Material and Methods

\section{Study design}

82156 consecutive sera submitted to the syphilis serology laboratory at the Clinic of Dermatology, 83 University Hospital of Zurich of patients at the Zurich University Hospital which were diagnosed between January 2008 and December 2010 having untreated syphilis were included in this retrospective study.

Routine testing included the VDRL test (Dade Behring, Düdingen, Germany), the TPPA test (Fujirebio Inc., Tokyo, Japan), the Fluorescent Treponemal Antibody-Absorption test (FTAABS, Biomérieux, Genève, Switzerland), and the Pathozyme Syphilis M Capture EIA (Omega Diagnostic Ltd., Alva, United Kingdom). After routine testing, sera were stored at $-20^{\circ} \mathrm{C}$ until further testing in 2011 with the Anti-Treponema-pallidum-ELISA (IgM; Euroimmun, Lübeck, Germany) and the recomWell Treponema IgM (Mikrogen, Neuried, Germany). To assess the specificity, 151 sera were tested including sera known to be potentially cross-reactive (anti-Borrelia burgdorferi $\operatorname{lgM}[n=10]$, anti-Epstein-Barr virus $\lg \mathrm{M}[n=10]$, antipatients $[n=20]$ and pregnant women $[n=20])$, negative sera without further specification $(n=$ $50)$ and sera of previously treated syphilis patients $(n=30)$.

\section{Patient characteristics}

Based on clinical data obtained from patients charts and serological results, patients were categorized as having primary syphilis $(n=59)$, secondary syphilis $(n=66)$, latent syphilis $(n=$ $25)$, or tertiary syphilis $(n=6)$ (Table 1 ). 50/59 primary syphilis patients had typical ulcers at ano-genital or oro-pharyngeal sites. The other nine patients had positive serology and erythematous lesions on the penis or the tonsils $(n=3)$ or a possible exposition to syphilis

104 without clinic $(n=6)$. Secondary syphilis patients had muco-cutaneous skin lesions typical for secondary syphilis. Patients with latent syphilis had positive serology but without clinical signs of 
syphilis. Patients were classified as having tertiary syphilis based on a combination of clinical and serological findings as well as CSF analysis

\section{Serological testing}

110 All tests were performed according to the manufacturer's instructions. To avoid false-negative

111 results due to the prozone phenomenon the VDRL was tested up to a 1:64 dilution.

112 The three EIAs are designed for the semi-quantitative detection of IgM antibodies against $T$.

113 pallidum. The Euroimmun Anti-Treponema-pallidum-ELISA (IgM) and the recomWell

114 Treponema IgM both involve indirect 'sandwich' EIA procedures using microtitration wells

115 coated with the recombinant treponemal antigens Tp15, Tp17, Tp47 and TmpA. After incubation

116 with the test serum, bound IgM antibodies are detected with an enzyme-labelled anti-human-

$117 \operatorname{Ig} \mathrm{M}$ antibody. For the Euroimmun Anti-Treponema-pallidum-ELISA (IgM), prior to incubation

118 with the test serum, interfering $\lg G$ antibodies are precipitated with anti-human-lgG antibodies.

119 Results are calculated as index values (optical density of sample/cut-off value) and are then

120 classified as negative $(<0.8)$, equivocal $(\geq 0.8,<1.1)$ or positive $(\geq 1.1)$.

121 According to the manufacturer's protocol for the recomWell a pre-adsorption of IgG antibodies

122 must not be done. However, due to moderate performance all samples were also tested with a

123 modified recomWell Treponema IgM protocol in which interfering IgG antibodies were

124 precipitated with Gullsorb reagent (Meridian Bioscience, Ohio, United States) containing anti-

125 human-IgG antibodies according to the manufacturer's protocol. For the recomWell Treponema

$126 \mathrm{IgM}$, results are calculated as antibody units per $\mathrm{ml}$ (optical density of sample/cut-off value $\times 20$ )

127 and are then classified as negative (<20), equivocal $(\geq 20, \leq 24)$ or positive $(>24)$.

128 In the Pathozyme Syphilis M Capture EIA microtitration wells are coated with anti-human

$129 \lg \mathrm{M}$ which captures IgM antibodies in the test serum onto the well. After washing a conjugate of

130 native T. pallidum antigen (Tp15, Tp17, Tp44 and Tp47) labelled with horseradish peroxidase is

131 applied. Results are calculated as index values (optical density of sample/cut-off value) and are

132 then classified as negative $(<0.9)$, equivocal $(\geq 0.9, \leq 1.1)$ or positive $(>1.1)$. 
134 was accepted as the final one. Thus, 27 positive sera and 5 control sera had to be repeated,

135 i.e., 14 Euroimmun, 6 recomWell, and 12 Pathozyme tests. In 10, 12, and 10 cases, respectively, results became negative, became positive, or remained equivocal upon repetition.

137 Repeated equivocal results were considered as positive for calculating sensitivity and 138 specificity.

\section{Statistical analysis}

141 Confidence intervals were calculated with GraphPad Prism, Version 5.04 (GraphPad Software, 142 Inc., La Jolla, California). Sensitivities were compared with the McNemar $X^{2}$ test $^{16}$ online at 143 http://graphpad.com/QuickCalcs (GraphPad Software, Inc., La Jolla, California last accessed 144 9th January 2013).

\section{Results}

148 Results of all tests are shown in Tables 2 and 3 and corresponding sensitivities and specificities are depicted in Table 4. In 148/156 patients the TPPA test was positive and in 8/156 it was equivocal/indeterminate. The VDRL test was positive or equivocal in 36/59 (61\%) patients with

151 primary syphilis, positive in 64/66 (97\%) patients with secondary syphilis, in 24/25 (96\%)

152 patients with latent syphilis and in all six patients with tertiary syphilis. VDRL and TPPA titres 153 were lower in primary syphilis compared to secondary, latent or tertiary syphilis (Table 2).

154 The IgM-specific EIAs started with low to moderate high scores in primary syphilis and 155 had a peak in secondary and to a lesser extent in tertiary syphilis, while in latent syphilis the 156 scores were lowest (Table 2). A total of $138(88.5 \%), 100(64.1)$, and $132(84.6 \%)$ of 156 sera of

157 syphilis patients were tested positive by the IgM-specific Pathozyme, recomWell, and

158 Euroimmun tests, respectively (Tables 2 and 4). As the recomWell Treponema IgM initially 159 showed moderate performance all specimens were retested with a modified protocol what 
resulted in a significantly (McNemar $\left.X^{2}=11.5, p<0.001\right)$ higher sensitivity $(73.7 \%$ as opposed to $64.1 \%)$.

In primary syphilis (Table 4), the sensitivities (\% sensitivity, 95\% Cl) of the Pathozyme $(89.8 \%, 79.2-96.2 \%)$ and the Euroimmun test (81.4\%, 69.1-90.3\%) were significantly higher than the sensitivities of the VDRL $(61 \%, 47.4-73.5 \%)$ and the modified recomWell test $(62.7 \%$, 49.2-75.0\%) as assessed by the McNemar $X^{2}$ test $\left(X^{2}>3.841, p<0.05\right)^{16}$. 23/59 primary syphilis patients had a negative VDRL. These patients probably had early infection as the TPPA titres (median 1:320, interquartile range $<1: 80-1: 320$ ) were low, too. In this group, IgM-specific Pathozyme, modified recomWell, and Euroimmun tests were positive in $22 / 23,10 / 23$, and $17 / 23$ cases, respectively. Detailed results of seven patients with indeterminate TPPA are summarized in Table 5; in all of them syphilis was confirmed with later control sera, i.e., TPPA became positive and IgM EIA tests decreased after therapy. It is therefore believed that they had very early infection. Pathozyme, modified recomWell, and Euroimmun tests were positive or equivocal in $7 / 7,2 / 7$ and $3 / 7$ cases, respectively.

The specificity of all tests ranged from $91.4-100 \%$ (Tables 3 and 4). All investigated specific IgM tests showed some cross-reactivity, especially the Euroimmun Anti-Treponemapallidum-ELISA had difficulties with sera containing IgM antibodies against human Herpes viruses (7/10 anti-Epstein-Barr virus IgM sera and 3/10 anti-Cytomegalovirus IgM sera).

\section{Discussion}

The clinical and laboratory diagnosis of primary syphilis can be difficult. Usually one or multiple, painless and indurate ulcers develop at the site of infection. However, the lesion may be atypical, ${ }^{1,17}$ e.g., i) only a papular lesion may develop, ii) it may be inconspicuous, iii) it does not develop in every case, iv) a syphilitic balanitis of Follman may be the only clinical expression. Patients might also seek medical advice after risk situations in the incubation period before development of clinical symptoms. Besides dark-field microscopy, which requires experienced 
187 staff and is labour-intensive, and molecular tests, which are not yet generally used and are 188 expensive, serological tests remain the most important tool for diagnosing syphilis. In the 189 present study, and in those of others, ${ }^{4,5}$ the TPPA was the most sensitive single test in all stages of disease, confirming its suitability as a screening test. ${ }^{1}$ The VDRL test sensitivity was

191 only $61 \%$ for primary syphilis, which is similar to what we found previously $(58 \%)^{2}$ but somewhat 192 lower than what other studies reported (66-87\%). ${ }^{3,18,19}$ The sensitivity of the IgM-specific syphilis

193 EIA assays for detecting primary syphilis was $89.8 \%$ for the Pathozyme Syphilis M Capture

194 (Omega Diagnostics) test and 81.4\% for the Anti-Treponema-pallidum-ELISA (IgM;

195 Euroimmun). This was significantly higher than $62.7 \%$ of the modified recomWell Treponema $196 \operatorname{IgM}$ (Mikrogen) or $61 \%$ of the VDRL test. In secondary syphilis which corresponds to the 197 hematogenic spreading of the pathogen throughout the entire body the EIAs had their highest 198 sensitivities, whereas in latent syphilis which clinically is a silent period IgM EIA values were low 199 and many samples were negative.

200 Most interestingly is the group of primary syphilis patients with negative VDRL. These $20123 / 59$ (39\%) cases all would have been missed when the VDRL was used as the only first-line 202 screening test as previously recommended in the United States. ${ }^{18,20}$ In this subgroup, the TPPA 203 was positive in 17/23 and indeterminate in 6/23 cases. TPPA alone would have allowed 204 diagnosing present or past syphilis infection in these cases (still, the six indeterminate cases 205 would have been doubtful requiring confirmation with second samples). However, the 206 combination of TPPA and IgM-specific EIA assays allowed correct diagnosis of early primary 207 syphilis in $22 / 23$ and $17 / 23$ cases with the Pathozyme and the Euroimmun assay, respectively. 208 The Pathozyme assay was even positive in seven cases with presumed very early infection with 209 some patients eventually still being in the incubation phase (Table 5). It has been previously 210 shown that a specific IgM EIA may be the only positive test in early infection. ${ }^{2,5}$ It is therefore 211 suggested that in cases of suspected early infection specific IgM EIAs should be used in 212 addition to other screening tests. ${ }^{1}$ It is important that clinicians communicate the suspicion of 213 early infection to the laboratory in order that an IgM EIA is utilized. 
Most previous studies investigating IgM-specific commercial assays have examined the

215 Mercia Syphilis M EIA (Microgen Products Ltd), ${ }^{4,5,8-12}$ in one study the Eti-syphilis-M was

216 analysed (DiaSorin). ${ }^{7}$ The reported sensitivities are similar to the ones found here, for primary

217 syphilis they were in the range of $82-94 \%$ and for secondary syphilis in the range of $60-100 \%$.

218 For latent syphilis the sensitivity was dependent on whether early (56-87\%) or late (0-50\%)

219 latent cases were examined. In one previous study the recomWell Treponema IgM assay was

220 investigated, ${ }^{13}$ the sensitivity in 19 primary syphilis cases was $89.5 \%$ which is higher than in the

221 present work. However, the assay was negative in all samples of later stages of syphilis.

222 Schmidt et al. ${ }^{10}$ evaluated nine commercially available EIAs with a panel of 52 highly selected

223 sera from primary syphilis patients, all negative with the microhemagglutination test for

224 T. pallidum. Eight assays were designed to detect IgG alone or in combination with IgM

225 antibodies. Most interestingly, the one assay detecting only IgM antibodies (Mercia Syphilis M

226 EIA) demonstrated the highest sensitivity (86.5\%) as compared to the other tests (22.6-76.9\%).

227 Remarkable is the fact that all these previous studies except two did not report the

228 specificity. ljsselmuiden et al. ${ }^{8}$ tested 48 samples of mainly treated syphilis patients and non-

229 infected neonates with a specificity of $98 \%$ and Sambri et al. ${ }^{13}$ tested 200 samples from blood-

230 donors and 60 samples from patients with possibly cross-reactive conditions (e.g. Lyme

231 disease, mononucleosis, pregnancy) with $100 \%$ specificity. In the present study, 151 sera

232 including 71 potentially cross-reactive samples were investigated for testing the specificity.

233 Except for the Euroimmun assay which had some difficulties with sera which were positive for

234 IgM antibodies against human Herpes viruses, all other assays showed specificities of 95.4-

$235100 \%$.

236 For monitoring the treatment response primarily non-treponemal tests such the VDRL or

237 the RPR are recommended. ${ }^{1,3}$ It has been shown previously that specific IgM EIAs are a reliable

238 supplement ${ }^{2,21,22}$ and this might be especially valuable in cases with an initially negative VDRL

239 test (mainly primary syphilis cases) and in case of a slow decline or persisting low VDRL

240 reactivity despite adequate therapy. However, it has to be noted that response rate may be

241 different among different commercial IgM EIAs and also different as compared to the VDRL. 
242 Studies which used the Mercia Syphilis M EIA reported negative test results one year after

243 treatment in $92-100 \%$ of patients presenting with early syphilis ${ }^{21,22}$ - as compared to $62-87 \%$ in a

244 study using the Pathozyme test. ${ }^{2}$

245 All three IgM tests are easy to perform and do not require special equipment or special

246 skills and knowledge of the staff. They have total incubation times of 2-2.5 hours and can be

247 executed routinely within half a day. The tests differ, however, in their accuracy with the

248 recomWell being the least sensitive. The recomWell assay is an indirect 'sandwich' EIA. In this

249 kind of assay patients $\lg G$ antibodies are usually precipitated with an anti-human $\lg G$ antibody

250 to avoid a competition with IgM antibodies in binding to the recombinant antigens what can

251 cause a false-negative result. This step is missing in the recomWell procedure. Therefore, a

252 modified recomWell protocol precipitating interfering IgG antibodies was tested. The sensitivity

253 thus significantly increased from 64.1 to $73.7 \%$. This demonstrates that the pre-adsorption of

254 interfering $\operatorname{lgG}$ antibodies increases the performance. With some optimization of the protocol the 255 sensitivity might be increased even more.

256 A limitation of this study is the fact that the sera were stored at $-20^{\circ} \mathrm{C}$ until testing with 257 the Euroimmun and the recomWell assay. It cannot be entirely excluded that this might have 258 influenced the result in some cases.

259 In summary, specific syphilis IgM EIA assays are highly sensitive in primary syphilis and 260 are specific. It is suggested that in cases of suspected early infection specific IgM EIAs should 261 be used in addition to other screening tests. Additionally, they allow differentiation of active and 262 past infection and may be used as supplement for monitoring treatment response. ElAs from 263 different manufacturers vary in their performance.

\section{Addendum}

267 During the course of this study Omega Diagnostics informed that the Pathozyme Syphilis M 268 Capture test is suspended from production until further notice. 


\section{Conflict of interest}

271 Mikrogen, Neuried, Germany and Pharma Consulting Marion Senn GmbH, Burgdorf,

272 Switzerland sponsored $50 \%$ and $25 \%$ of costs of the recomWell Treponema IgM (Mikrogen)

273 tests, respectively; Euroimmun Schweiz AG, Luzern, Switzerland has sponsored all Euroimmun

274 Anti-Treponema-pallidum-ELISA (IgM) tests. The author has served as speaker for Euroimmun

275 Switzerland and has received travel grants from Euroimmun Switzerland. No company had

276 influence on the design of the study, interpretation of results, the final manuscript or any other

277 part of the study.

278

\section{Acknowledgments}

280 I deeply appreciate the technical assistance of Tamara Eicher. I thank Thomas Kündig for

281 critical review of the manuscript, Nicole Graf for statistical advice, and Walter Bossart and

282 Reinhard Zbinden for providing potentially cross-reactive serum samples.

283

284

285

286 


\section{References}

288 1. French P, Gomberg M, Janier M, Schmidt B, van Voorst Vader P, Young H. IUSTI: 2008

European guidelines on the management of syphilis. Int J STD AIDS 2009;20(5):300-9.

2. Fröhlich Knaute D, Graf N, Lautenschlager S, Weber R, Bosshard PP. Serological response to treatment of syphilis according to disease stage and HIV status. Clin Infect Dis 2012;doi: 10.1093/cid/cis757

3. Larsen SA, Steiner BM, Rudolph AH. Laboratory diagnosis and interpretation of tests for syphilis. Clin Microbiol Rev 1995;8(1):1-21.

4. Manavi $\mathrm{K}$, Young $\mathrm{H}$, McMillan A. The sensitivity of syphilis assays in detecting different stages of early syphilis. Int J STD AIDS 2006;17(11):768-71.

5. McMillan A, Young $H$. Qualitative and quantitative aspects of the serological diagnosis of early syphilis. Int J STD AIDS 2008;19(9):620-4.

6. Shannon R, Booth SD. The pattern of immunological responses at various stages of syphilis. Br J Vener Dis 1977;53(5):281-6.

7. Castro R, Prieto ES, Santo I, Azevedo J, Exposto Fda L. Evaluation of an enzyme immunoassay technique for detection of antibodies against Treponema pallidum. J Clin Microbiol 2003;41(1):250-3.

8. Ijsselmuiden OE, van der Sluis JJ, Mulder A, Stolz E, Bolton KP, van Eijk RV. An IgM capture enzyme linked immunosorbent assay to detect IgM antibodies to treponemes in patients with syphilis. Genitourin Med 1989;65(2):79-83.

9. Lefevre JC, Bertrand MA, Bauriaud R. Evaluation of the Captia enzyme immunoassays for detection of immunoglobulins $\mathrm{G}$ and $\mathrm{M}$ to Treponema pallidum in syphilis. J Clin Microbiol 1990;28(8):1704-7.

10. Schmidt BL, Edjlalipour M, Luger A. Comparative evaluation of nine different enzymelinked immunosorbent assays for determination of antibodies against Treponema pallidum in patients with primary syphilis. J Clin Microbiol 2000;38(3):1279-82.

11. Young H, Pryde J, Duncan L, Dave J. The Architect Syphilis assay for antibodies to Treponema pallidum: an automated screening assay with high sensitivity in primary syphilis. Sex Transm Infect 2009;85(1):19-23.

12. Tabidze IL, Lee FK, Tambe P, Rocha E, Larsen SA, Stoll BJ, et al. Enzyme-linked immunospot assay for the diagnosis of active Treponema pallidum infection during the various stages of syphilis. Sex Transm Dis 1999;26(8):426-30.

13. Sambri V, Marangoni A, Simone MA, D'Antuono A, Negosanti M, Cevenini R. Evaluation of recomWell Treponema, a novel recombinant antigen-based enzyme-linked immunosorbent assay for the diagnosis of syphilis. Clin Microbiol Infect 2001;7(4):200-5.

14. Rawstron SA, Mehta S, Bromberg K. Evaluation of a Treponema pallidum-specific IgM enzyme immunoassay and Treponema pallidum western blot antibody detection in the diagnosis of maternal and congenital syphilis. Sex Transm Dis 2004;31(2):123-6.

15. Wang LN, Li JM. Evaluation of immunoglobulin M and G Western blot and ELISA for screening antibodies to Treponema pallidum in blood donors. Sex Transm Dis 2009;36(7):413-6.

16. Linnet K, Bossuyt PM, Moons KG, Reitsma JB. Quantifying the accuracy of a diagnostic test or marker. Clin Chem 2012;58(9):1292-301.

17. Tramont EC. Treponema pallidum (Syphilis). In: Mandell GL, Bennett JE, Dolin R, editors. Principles and practice of infectious diseases. 6th ed. Philadelphia: Elsevier, 2005:2768-85.

18. Creegan L, Bauer HM, Samuel MC, Klausner J, Liska S, Bolan G. An evaluation of the relative sensitivities of the venereal disease research laboratory test and the Treponema pallidum particle agglutination test among patients diagnosed with primary syphilis. Sex Transm Dis 2007;34(12):1016-8.

19. Janier M, Chastang C, Spindler E, Strazzi S, Rabian C, Marcelli A, et al. A prospective study of the influence of HIV status on the seroreversion of serological tests for syphilis. Dermatology 1999;198(4):362-9. 
20. Sena AC, White BL, Sparling PF. Novel Treponema pallidum serologic tests: a paradigm shift in syphilis screening for the 21st century. Clin Infect Dis 2010;51(6):700-8.

21. McMillan A, Young H. Reactivity in the Venereal Diseases Research Laboratory test and the Mercia IgM enzyme immunoassay after treatment of early syphilis. Int J STD AIDS 2008;19(10):689-93.

22. Rotty J, Anderson D, Garcia M, Diaz J, Van de Waarsenburg S, Howard T, et al.

Preliminary assessment of Treponema pallidum-specific IgM antibody detection and a new rapid point-of-care assay for the diagnosis of syphilis in human immunodeficiency virus-1-infected patients. Int J STD AIDS 2010;21(11):758-64. 